\title{
Treatment of intrabony defects with modified perforated membranes in aggressive periodontitis: a 4-year follow-up of a randomized controlled trial
}

\author{
Bartłomiej Górski ${ }^{1}$ (D) Stanisław Jalowski ${ }^{2} \cdot$ Renata Górska $^{1}$ • Maciej Zaremba ${ }^{1}$
}

Received: 5 March 2019 / Accepted: 20 June 2019 / Published online: 19 July 2019

(C) The Author(s) 2019

\begin{abstract}
Objective (1) To assess long-term outcomes 4 years following guided tissue regeneration (GTR) of intrabony defects in patients diagnosed with aggressive periodontitis (AgP) and (2) to identify predictors of clinical attachment level (CAL) gain and bone/ graft density gain.

Materials and methods In 15 patients, two deep intrabony defects were randomly treated with xenogenic graft plus modified perforated membranes (MPM, tests) or xenogenic graft plus standard collagen membranes (CM, controls). After 4 years, clinical and radiographic outcomes were evaluated and compared with outcomes at baseline and after 1 year.

Results After 4 years, 14 test sites and 13 control sites were available for analysis. One tooth was lost as a result of root fracture. There were significant improvements in all evaluated parameters after 1 and 4 years in relation to baseline, but no differences were observed between tests and controls. However, some non-significant changes were found between 1 and 4 years. Regression analyses showed that recurrence of periodontitis was a significant predictor for CAL gain $(p=0.001)$ and bone/ graft density gain $(p=0.024)$ from 1 to 4 years.

Conclusions GTR of intrabony defects in AgP with either standard or modified CM yielded similarly successful and maintainable clinical benefits for compromised teeth 4 years following the surgery. The use of MPM showed no additional benefit.

Clinical relevance This study demonstrates that most of the positive outcomes of GTR in AgP may be preserved over 4 years. Periodontitis recurrence might influence long-term outcomes.
\end{abstract}

Keywords Aggressive periodontitis · Collagen membrane · Guided tissue regeneration · Intrabony defect · Long-term outcomes

\section{Introduction}

The factors impinging on regeneration of periodontal defects are rather intricate, and the appropriate type and an adequate number of cells, suitable scaffolding materials, and a beneficial microenvironment with signaling molecules are of utmost importance. Mesenchymal stem/progenitor cells (MSCs) are of special interest in periodontal wound healing since they are most likely the parental cells of synthetic cells including

Bartłomiej Górski

bartek_g3@tlen.pl

1 Department of Periodontology and Oral Mucosa Diseases, Medical University of Warsaw, Miodowa St 18, 00-246 Warsaw, Poland

2 Department of Dental and Maxillofacial Radiology, Medical University of Warsaw, Nowogrodzka St 59, 02-006 Warsaw, Poland osteoblasts, cementoblasts, and fibroblasts, accounting for the restoration of lost periodontal tissues. Undifferentiated MSCs reside in the periosteum, endosteum, marrow spaces, periodontal ligament, and tooth-surrounding soft tissues. The value of periosteum and gingiva, as a prominent source of MSCs, which may potentially enhance regeneration of intrabony defects, has lately been emphasized. Periosteum-derived stem/ progenitor cells (PDPCs) and gingival mesenchymal stem/ progenitor cells (GMSCs) promoted angiogenesis and showed unique anti-inflammatory and immunomodulatory functions, as well as osteogenic potential [1-4]. It is worth to note that GMSCs were isolated from healthy and periodontally diseased tissues and exhibited high mineralization, as well as strong expression of alkaline phosphatase in 3D culture on polycaprolactone (PCL) scaffolds [1]. Moreover, under suboptimal proliferation conditions (incubation with TNF- $\alpha$ and Il$1 \beta)$, GMSCs displayed a higher proliferation potential than periodontal ligament-derived MSCs [3]. All of the 
abovementioned suggest that these cells may be involved in homeostasis and regeneration of the periodontium.

A great variety of techniques and biomaterials have been introduced to restore the unique architecture and function of damaged periodontal tissues, but periodontal regeneration has not been fully accomplished with recognized therapeutic strategies [5]. Guided tissue regeneration (GTR) may promote the formation of new cementum, alveolar bone, and functionally oriented periodontal ligament, thus effectively reducing periodontitis-caused tooth loss [6]. In this technique, a biocompatible barrier membrane is placed between a surgical flap and root surface in order to guide cell recruitment in a selective manner. The use of occlusive membrane prevents epithelial downgrowth and fibroblast transgrowth into the wound space, as well as allows participation of MSCs deriving from marrow spaces, endosteum, periodontal ligament, and perivascular tissues in the regenerative processes. On the other hand, barrier placement excludes any contribution of PDPCs and GMSCs to healing processes taking place in intrabony defects. Moreover, the occlusive membrane may hinder the penetration of growth-regulatory factors into the barrierprotected area [7]. Generally speaking, barrier membranes denude the wound site from the regenerative potential of the periosteum [8]. With that knowledge in mind, some scientists endeavored to evaluate the effects of membrane perforations on clinical outcomes of GTR, as it was also reported that complete tissue occlusion was not a critical determinant for bone regeneration [9-11]. The rationale behind this idea was that pores in the membrane would permit undifferentiated MSCs residing in the periosteum and gingival connective tissue, as well as growth factors to migrate into intrabony defect and contribute to regenerative processes. It was later found that macro-membrane perforations of $0.2,0.4$, and $0.7 \mathrm{~mm}$ allowed for cellular migration and preserved membrane stiffness [12]. Furthermore, analysis of the gingival crevicular fluid in sites treated with perforated collagen membranes displayed elevated concentration of bone morphogenetic protein-2 (BMP-2), platelet-derived growth factor-BB (PDGF-BB), and vascular endothelial growth factor (VEGF), which improved the clinical results of periodontal regeneration $[13,14]$. It has also been implied that owing to mechanical interlocking of fibrin strands with the pores, the formed fibrin clot might be better stabilized. According to everything mentioned above, cell-permeable membranes, which allowed more physiologic interplays between different elements of periodontium, have emerged as a novel therapeutic approach. Recent clinical investigations demonstrated significant new bone formation and increased bone density in intrabony defects treated with perforated collagen membranes up to 12 months postoperatively, in comparison with standard collagen membranes, but the long-term outcomes have not been reported $[9,15,16]$.
A large number of well-designed randomized, controlled trials (RCTs) have highlighted promising results of GTR in patients diagnosed with chronic periodontitis $(\mathrm{ChP})$, but only a few prospective studies evaluated aggressive periodontitis (AgP) patients [17]. As a matter of fact, Corbella et al. [18] recently conducted a systematic review on periodontal regeneration procedures in individuals affected by $\mathrm{AgP}$ and concluded that periodontal regenerative surgery might be successfully carried out in those individuals. However, the level of evidence of the papers included in this review was considered low, as data have mostly been drawn from uncontrolled studies and short follow-ups, and only three papers reported on two RCT, which limited the possibility of performing metaanalysis $[19,21,29]$. The other important facet related to the included studies was a strong heterogeneity observed among them. The follow-up period of these RCTs varied from 6 to 12 months. What is more, no RCT described a sample-size calculation procedure before enrollment, hereby restricting the possibility to assess statistical power of the outcomes (type II error). Consequently, there is no consensus regarding longtime efficacy of regenerative procedures in AgP patients. Accordingly, there is still a pressing need for more highquality data to evaluate whether and how long the treatment benefits of GTR can be maintained [22].

To the best of our knowledge, no RCT exists with respect to long-term outcomes of regenerative periodontal therapy in patients with AgP. Thus, the main objective of this study was to assess 4-year results of GTR of deep intrabony defects with modified perforated collagen membranes (MPM) in AgP patients. Additionally, the secondary objective was to detect predictors for clinical attachment level (CAL) gain and bone/ graft density gain in digital subtraction radiography (DSR) in the long term.

\section{Materials and methods}

\section{Study design}

This 4-year follow-up of a double-blinded, split-mouth, RCT compares two regenerative treatment modalities of intrabony defects in AgP patients. The test group was treated with deproteinized bovine bone mineral (DBBM) plus modified perforated membranes (MPM), and the control group was treated with DBBM plus standard collagen membranes (CM). The design of the trial has been thoroughly described along with the 1 -year results $[15,16]$. Participants of the original study were longitudinally followed for 4 years (Fig. 1). The study was carried out in accordance with the Helsinki Declaration of 1975, as revised in Tokyo in 2004 after approval of the study design by the Bioethics Committee (KB/135/ 2014). Patients were informed of the nature, potential risks, 
Fig. 1 Consort diagram showing the study outline

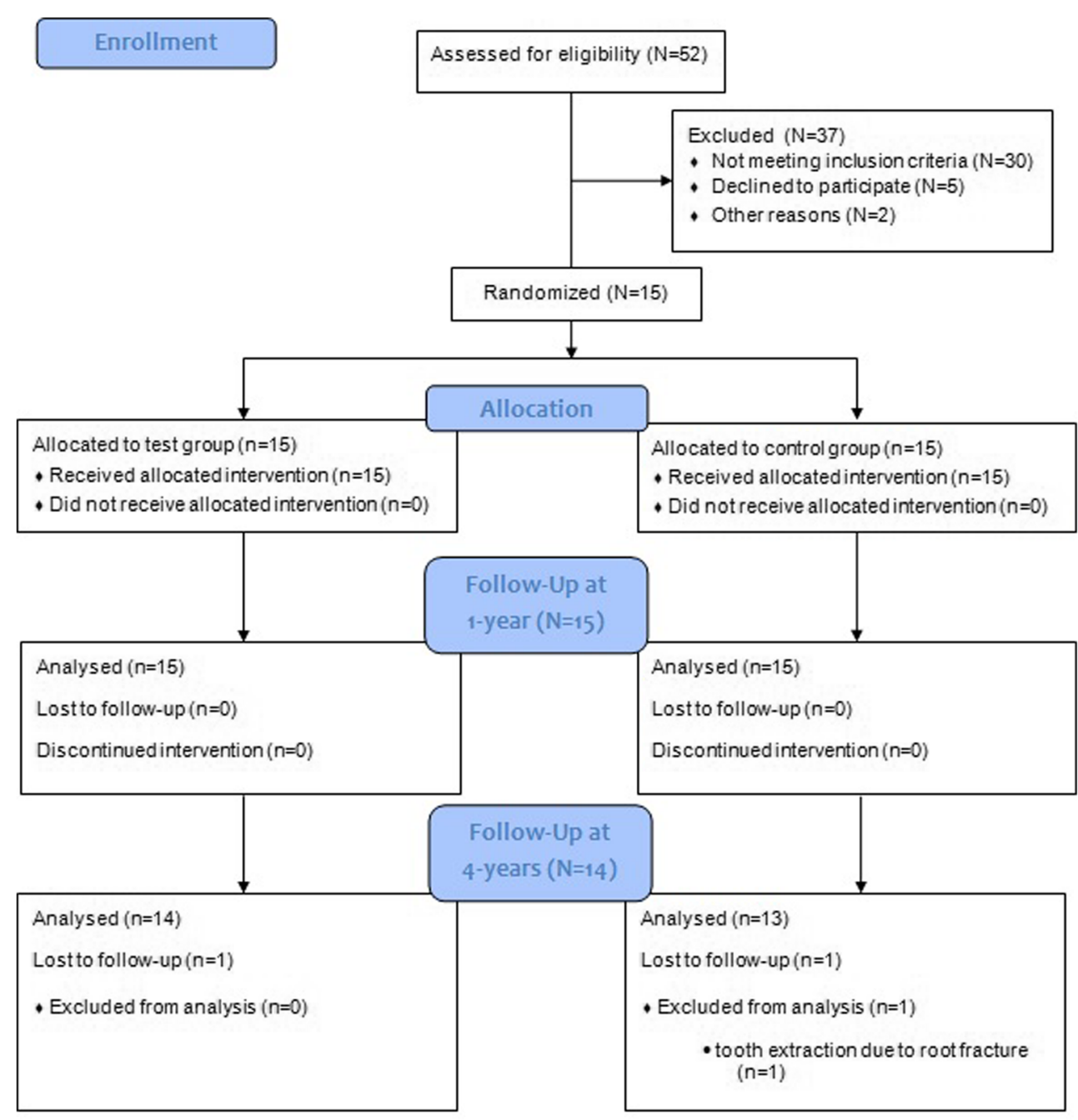

$\mathrm{N}$-number of patients, $\mathrm{n}$ - number of defects and benefits of their participation in the study. All participants signed consent forms.

\section{Subject population and sample size calculation}

Following fulfillment of non-surgical periodontal treatment consisting of scaling and root debridement with additional antibiotic therapy (amoxicillin $500 \mathrm{mg}+$ metronidazole $250 \mathrm{mg}$ three times daily for 1 week), 15 patients (10 women and 5 men) aged 22-49 years (mean age $37.9 \pm 7.95$ ) in good general health were signed up for this RCT. The patients were reevaluated at 6 weeks. Two comparative intrabony defects from each subject were selected. Baseline intra-oral radiographs were taken 1 day before the surgery, while clinical measurements were made on the day of the surgery.

According to a study by Gamal and Iacono [9], using MPM in GTR of intrabony defects may lead to additional CAL gain of approximately $1.2 \mathrm{~mm}$. Therefore, the sample size calculation determined that 8 subjects per treatment group would provide $80 \%$ power to disclose a true difference of $1.2 \mathrm{~mm}$ between test and control, assuming 0.05 as the level of significance and $1.0 \mathrm{~mm}$ as the common standard deviation (SD). Having in mind that some patients might be lost during follow-up, 15 subjects were enrolled.

\section{Inclusion and exclusion criteria}

Inclusion criteria were as follows: (1) diagnosis of $\mathrm{AgP}$ according to definitions of AAP of 1999 [23]; (2) no systemic diseases; (3) no use of medications affecting periodontal status; (4) non-smokers; (5) no pregnant or lactating; (6) history of periodontitis in parents or siblings; (7) presence of at least two teeth with PPD $\geq 6 \mathrm{~mm}, \mathrm{CAL} \geq 5 \mathrm{~mm}$, and defect depth (DD) $\geq 3 \mathrm{~mm}$ as detected in periapical radiographs; (8) fullmouth plaque score (FMPS) $\leq 20 \%$; (9) full-mouth bleeding score $($ FMBS $) \leq 20 \%$; (10) tooth had to be vital or properly treated; (11) no furcation involvement; (12) the width of keratinized tissue on the vestibular site of the tooth $\geq 2 \mathrm{~mm}$. 


\section{Clinical measurements}

Clinical measurements were taken by the same experienced and calibrated examiner (MZ), who was masked with respect to treatment. FMPS was calculated as the percentage of tooth surfaces that exhibited plaque [24] and FMBS as the percentage of periodontal pockets that bled from the bottom $15 \mathrm{~s}$ after careful probing [25]. Clinical parameters were registered at six points of each tooth (i.e., distobuccal, buccal, mesiobuccal, distolingual, lingual, mesiolingual) with a graded periodontal probe (UNC probe $15 \mathrm{~mm}$, Hu-Friedy, Chicago, IL, USA), and rounded off to the nearest millimeter: (1) PPD as the distance from the gingival margin to the base of periodontal pocket; (2) CAL as the distance from the cemento-enamel junction (CEJ) to the base of periodontal pocket; (3) gingival recession (GR) as the distance from CEJ to the gingival margin. Clinical measurements were made immediately before as well as 1 and 4 years after periodontal surgery.

Intrasurgery measurements were recorded upon completion of intrasurgical debridement: (1) defect depth as the distance between bottom of the defect and the most coronal point of the bony walls surrounding the defect; (2) defect width as the distance from the most coronal point of the bony walls surrounding the defect to the root surface; (3) the number of remaining walls of the defect (defects were classified as onewall, two-wall, and three-wall defects).

\section{Radiographic measurements}

Radiographic examination of digital intra-oral radiographs taken before surgery, after 1 year, and after 4 years using the paralleling technique was carried out by an experienced and calibrated clinician (SJ) who was masked with respect to the surgical intervention. Standardized reproducible periapical radiographs were collected from each site with phosphor plates (KaVo Scan eXam, KaVo, Biberach, Germany) with modified, individual film holders and paralleling technique using an x-ray unit operating at $70 \mathrm{kV}, 4 \mathrm{~mA}$, and 0.1 -s exposure time, prior to surgery and at 12 and 48 months postoperatively. DD was calculated as the distance between the bottom of the defect (BD) and the alveolar crest. Defect angle was measured between the intersection of CEJ-BD line and the delimitation of the wall of the defect [26]. Linear defect fill (LDF) was calculated by subtracting CEJ-BD distance at the end of 4 years from CEJ-BD at either baseline or at 1 year postoperatively, while percentage defect fill (\%DF) by dividing LDF by DD at evaluated time [27]. Designated software was used for linear measurements (Planmeca Romexis Viewer software, Planmeca, Helsinki, Finland). Radiographs obtained at 1 year and 4 years were subtracted from the radiographs taken at baseline, and radiographs taken at 4 years were subtracted from the radiographs taken at 1 year with Image $®$ software (Research Services Branch, NIH, Bethesda, MD, USA), as previously described in details [16]. The areas that showed gain in radiographic density (lightened areas) were measured in percentages.

\section{Randomization and surgical procedures}

Allocation of treatment sites was carried out using a computerized random number generator by a person not involved in the study (SJ) before surgery and concealed in sealed and opaque envelopes. All defects were treated by one surgeon (BG) with the modified papilla preservation technique (MPPT) in large interdental spaces (the width of interdental space $>2 \mathrm{~mm}$ ) or with the simplified papilla preservation flap (SPPF) in narrow interdental spaces (the width of interdental space $2 \mathrm{~mm}$ or less) $[28,29]$. DBBM (Bio-Oss $®$, Geistlich Biomaterials, Princeton, NJ, USA) were placed to fill the intrabony defects. It was only at this stage that a sealed envelope was opened, and treatment allocation was revealed to the surgeon. Patients were not informed about treatment allocation. In the test group, defects were covered with modified perforated porcine collagen membrane (MPM/test, BioGide ${ }^{\circledR}$, Geistlich Biomaterials), whereas in the control group with standard collagen membrane ( $\mathrm{CM} /$ control, Bio-Gide $\left.{ }^{\circledR}\right)$. Membrane perforations were made by using a custom-made acrylic template and a hand-spreader number 40 (Poldent, Warsaw, Poland) at a distance of $2 \mathrm{~mm}$ throughout the length of the membrane, leaving a coronal occlusive rim of $\sim 2$ $3 \mathrm{~mm}$ [9]. Perforation diameters varied approximately from about 0.46 to $0.72 \mathrm{~mm}$. Subsequently, membranes were trimmed to cover the defect and $2-3 \mathrm{~mm}$ of remaining bone and adapted in line with the protocol of the manufacturer without suturing. Primary soft tissue closure was achieved with sutures.

\section{Post-surgical period and long-term supportive periodontal care}

Patients were requested to rinse with $0.2 \%$ chlorhexidine digluconate mouth rinse three times per day for 3 weeks. Healing was uneventful in all subjects. At week 2, sutures were removed and patients resumed careful brushing with a soft toothbrush. In five patients (3 MPM sites and $2 \mathrm{CM}$ sites), membrane exposure was observed at 2 to 3 weeks after surgery. Exposed areas were irrigated with $0.2 \%$ chlorhexidine solution at the follow-up appointments and with daily application of $1 \%$ chlorhexidine gel by patients up to the time of complete re-epithelialization. All patients were placed on a 2week recall system for 3 months, and every 3 months for 1 year. Each session consisted of reinforcement of oral hygiene instructions and supragingival plaque removal. After 1-year follow-up, patients were scheduled for supportive periodontal therapy (SPT) with recall intervals varied between 3 and 6 months. The participation of patients in SPT was 
recorded. The recurrence of periodontitis was considered if $>$ $30 \%$ of patient's teeth needed to be re-instrumented subgingivally (PPD $\geq 5 \mathrm{~mm}$ ) [30]. These sites were treated with non-surgical root debridement.

\section{Data analysis}

Statistical analysis was carried out using Statistica 13 (Dell Inc. (2016); Dell Statistica (data analysis software system), version 13; software.dell.com). Normality of distribution was assessed using Shapiro-Wilk test and by visual inspection of histograms. For quantitative variables with normal distribution, mean \pm SD (95\% confidence interval) were given. The primary outcome variable was CAL gain at 4 years, and the secondary variables were changes in bone density, PPD reduction, and \%DF at 4 years. For statistical analysis, the measurements at the site with the greatest presurgical CAL value were used. By deducting the 4-year values from either the baseline values or 1-year values, the 4-year changes in clinical and radiographic outcomes were calculated. Consequently, a positive change implied a reduction in PPD, a gain in CAL, a decrease in GR, and a reduction in DD. Comparisons between MPM-treated and CM-treated sites at the same time points were performed using $t$ test for independent variables, while comparisons of changes in time within the same group were evaluated by $t$ test for paired data. Multiple linear regression was used to evaluate the relationship of age, sex, tooth type, surgical procedure, endodontic status, FMPS, FMBS, PPD, CAL, DD, RVG angle at 1 year, patients' compliance, and periodontitis recurrence with CAL gain and bone/graft density gain from 1 year to 4 years postoperatively (dependent variables). The model quality was assessed by means of residual analysis on linearity and homoscedasticity. Any $p$ values of less than $0.05(p<0.05)$ were considered statistically significant.

\section{Results}

The baseline description of defects showed no statistically significant differences between tests and controls (Table 1). All 15 recruited patients who received the intended treatment complied up to 1 year. Between 1 and 4 years, there was 1 drop-out (patient could not be traced anymore). The remaining 14 patients were 10 women and 4 men, among whom $9(64 \%)$ participated regularly in professional SPT, and $5(36 \%)$ received no SPT. One patient had one tooth extracted due to root fracture (CM-treated site). Consequently, 14 teeth in test sites and 13 teeth in control sites were suitable for analysis after 4 years (Fig. 1). Four (28\%) patients showed periodontitis recurrence, among whom $2(22 \%)$ participated regularly in SPT program, whereas the other $2(40 \%)$ did not.
The mean FMPS increased from $8 \%$ at baseline to $19 \%$ after 1 year and $26 \%$ after 4 years, while the mean FMBS raised from $12 \%$ at baseline to $19 \%$ after 1 year and $27 \%$ after 4 years. The mean PPD at baseline was found to be $7.4 \mathrm{~mm}$ in test sites and $7.2 \mathrm{~mm}$ in control sites and declined to $3.4 \mathrm{~mm}$ and $3.7 \mathrm{~mm}$ after 1 year, respectively. After 4 years, the mean PPD increased again by $0.2 \mathrm{~mm}$ in both groups. The mean CAL started at baseline at $8.7 \mathrm{~mm}$ (MPM-treated sites) or $8.5 \mathrm{~mm}$ (CM-treated sites) and decreased to $4.0 \mathrm{~mm}$ and $4.2 \mathrm{~mm}$ after 1 year, respectively. Twenty-eight percent of test sites (4 defects) demonstrated CAL loss from 1 to 4 years, $43 \%$ (6 defects) showed CAL gain, and in 28\% (4 sites) CAL did not change; the respective values for control sites were: $31 \%$ (4), 31\% (4), and 38\% (5). A mean baseline DD in MPM-treated sites was $5.9 \mathrm{~mm}$ and in CM-treated sites $5.3 \mathrm{~mm}$. After 1 year, mean DD decreased by $5.2 \mathrm{~mm}$ in MPM-treated sites, and by $4.4 \mathrm{~mm}$ in CM-treated sites. After 4 years, the measured mean DD reached $0.5 \mathrm{~mm}$ in test sites and $0.7 \mathrm{~mm}$ in control sites (Figs. 2 and 3). The mean $\%$ DF after 1 year was found to be $80 \%$ in the test and $81.7 \%$ in the control group and increased to $87 \%$ in the test and to $84.1 \%$ in the control group after 4 years. Constant increase in bone/graft density was observed in MPM-treated sites that reached $88 \%$ after 1 year and increased by mean $0.5 \%$ after 4 years. In CM-treated sites, mean bone/graft density increased by $82 \%$ after 1 year and declined by $0.01 \%$ after 4 years. No significant differences were observed between test and control sites. The thorough results of clinical and radiographic outcomes are presented in Tables 2 and 3. Table 4 depicts the frequency distribution of sites with different PPD for tests and controls at baseline, as well as after 1 and 4 years.

Table 5 depicts the results of regression analysis with CAL gain from 1 to 4 years postoperatively, as the dependent variable. The data show no multicollinearity. Periodontitis recurrence was the only significant predictor for CAL gain ( $p=$ 0.003 ), and the recurrence of periodontitis resulted in $2.9 \mathrm{~mm}$ CAL loss. Almost $80 \%$ of the variability could be explained by the regression model $\left(R^{2}=0.799\right)$. An analogous analysis was carried out with bone/graft density gain (Table 6). The data show no multicollinearity. Quite similarly, periodontitis recurrence was the only significant predictor for bone density gain $(p=0.024)$. The recurrence of periodontitis decreased bone/graft density gain by $9.7 \%$. Sixty-five percent of the variability could be explained by the regression model $\left(R^{2}=\right.$ $0.653)$. Both model qualities were satisfying given the linear relationship and homoscedasticity of the residuals (Fig. 4).

\section{Discussion}

Definitions and classifications of periodontal diseases have been subject to substantial discussion for many decades. The 1999 International Workshop on Classification of Periodontal 
Table 1 Baseline clinical characteristics: tooth type, tooth position, radiographic angle (RVG angle), intrasurgical measurements, and defect morphology (mean with $95 \%$ confidence interval and standard deviation)

Fig. 2 Treatment of intrabony defect on distal surface of tooth 34 utilizing modified perforated membrane (test site). a Preoperative 7-mm probing pocket depth (PPD). b Intrabony defect after completion of intrasurgical debridement. c Trimmed and perforated collagen membrane. $\mathbf{d}$ Membrane positioned over intrabony defect filled with xenogenic graft. e Wound closure sutures. f Clinical photo, 2 weeks post-surgery. g Clinical photo, 1 year post-surgery showing PPD of $4 \mathrm{~mm}$. h Clinical photo, 4 years post-surgery showing PPD of $4 \mathrm{~mm}$. i Baseline radiograph demonstrates intrabony defect. j Radiograph at 1 year post-surgery shows bone filling. $\mathbf{k}$ Radiograph at 4 years post-surgery shows stable outcome

\begin{tabular}{llll}
\hline Variables & Test sites $(n=15)$ & Control sites $(n=15)$ & $p$ value \\
\hline Tooth type $(n)$ & & 7 & \\
$\quad$ Molars & 7 & 3 & \\
Premolars & 4 & 5 & \\
$\quad$ Upper incisors, canines & 4 & 7 & 0.303 \\
Tooth position $(n)$ & & 8 & 0.725 \\
Maxillary teeth & 5 & $26.0[22.2-29.9] \pm 7.0$ & 0.725 \\
Mandibular teeth & 10 & & \\
Radiographic angle (degrees) & $23.24[21.4-25.5] \pm 3.8$ & $5.3[4.1-6.6] \pm 2.3$ & \\
Intrasurgical measurements $(\mathrm{mm})$ & & $2.9[2.5-3.3] \pm 0.7$ & \\
Defect depth & $5.5[4.7-6.4] \pm 1.6$ & & \\
Defect width & $3.5[2.6-4.4] \pm 1.6$ & 3 & \\
Defect morphology $(n)$ & 2 & 4 & \\
One-wall & 4 & & \\
Two-wall & 9 & & \\
Three-wall & & & \\
\hline
\end{tabular}

$n$ number of split-mouth defects
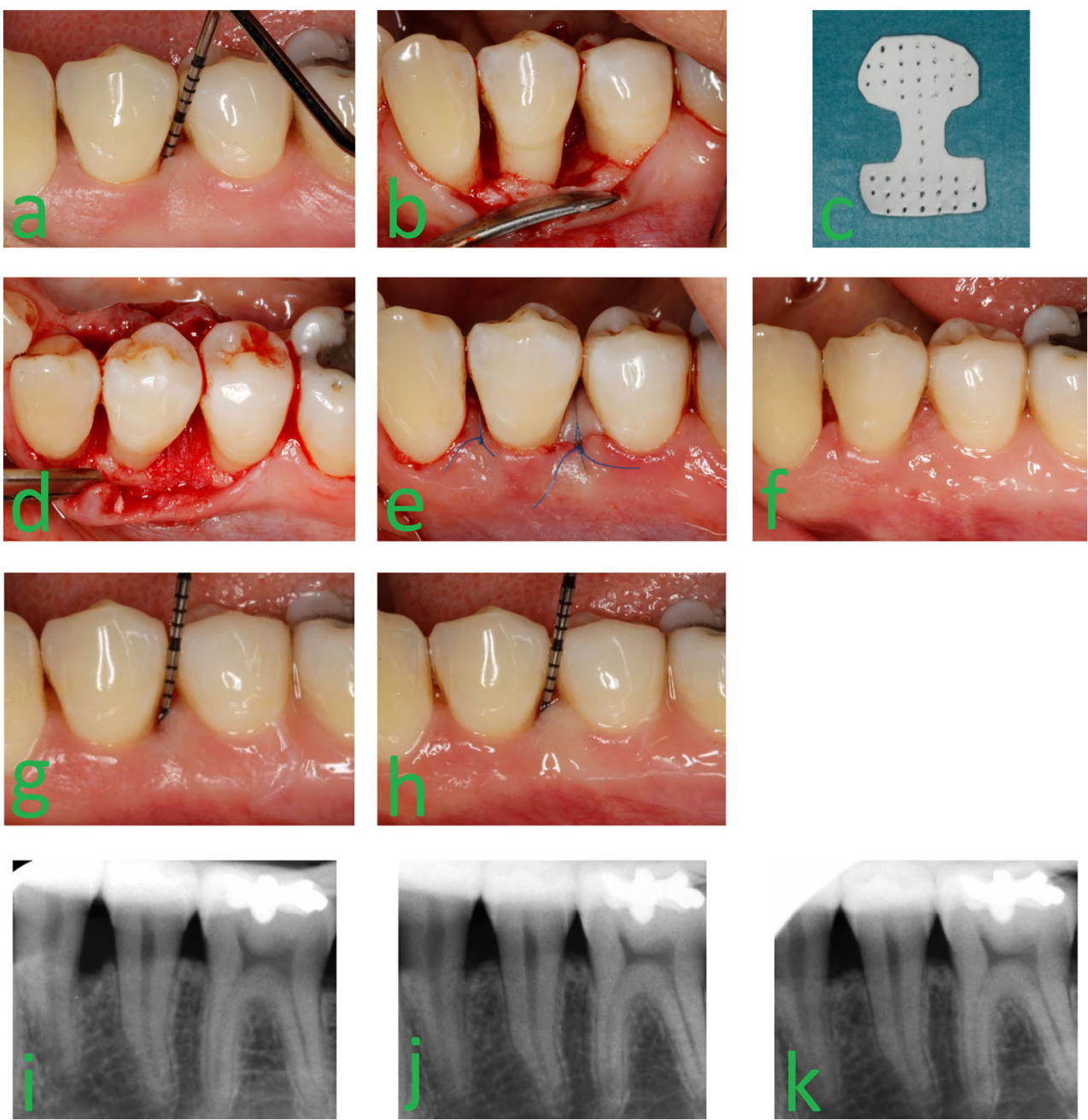
Fig. 3 Treatment of intrabony defect on distal surface of tooth 44 utilizing standard collagen membrane (control site). a Preoperative 7-mm probing pocket depth (PPD). b Intrabony defect after completion of intrasurgical debridement. c Trimmed collagen membrane. d Membrane positioned over intrabony defect filled with xenogenic graft. e Wound closure sutures. f Clinical photo, 2 weeks post-surgery. g Clinical photo, 1 year post-surgery showing PPD of $4 \mathrm{~mm}$. h Clinical photo, 4 years post-surgery showing PPD of $4 \mathrm{~mm}$. i Baseline radiograph demonstrates intrabony defect. $\mathbf{j}$ Radiograph at 1 year post-surgery shows bone filling. $\mathbf{k}$ Radiograph at 4 years post-surgery shows stable outcome
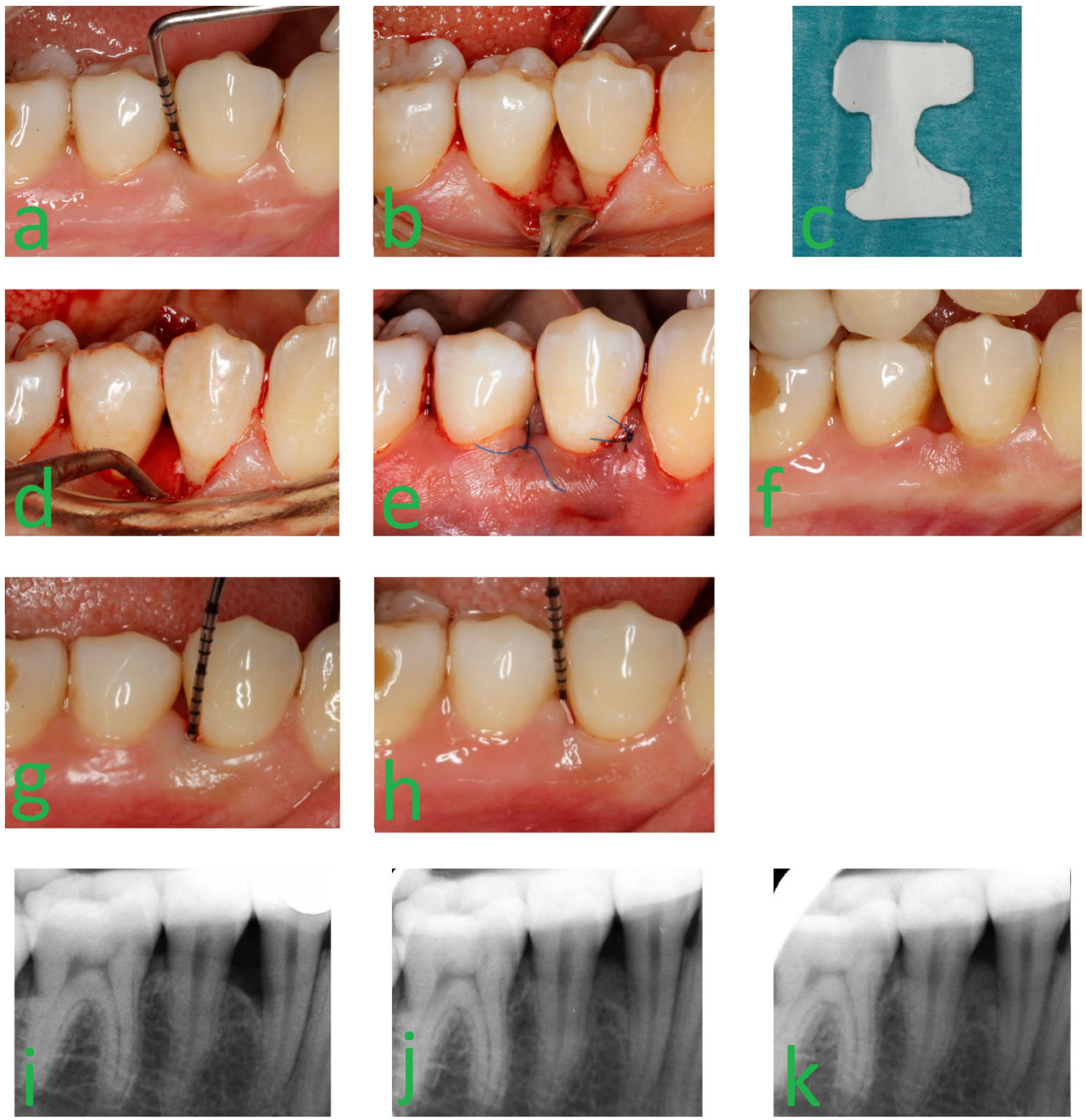

Diseases distinguished between $\mathrm{AgP}$ and $\mathrm{ChP}$ [31]. Accordingly, AgP was defined as a rare inflammatory condition characterized by rapid and severe destruction of attachment and bone with minimal presence of microbial deposits that affected younger individuals with familial aggregation. The prevalence of $\mathrm{AgP}$ was estimated to vary from 0.1 to $1 \%$ in European Caucasians [32, 33]. This system has been in use for the last 19 years. However, the 2017 World Workshop on Classification of Periodontal and Peri-Implant Diseases grouped $\mathrm{AgP}$ and $\mathrm{ChP}$ under a single category, periodontitis, since no solid evidence was found to consider $\mathrm{AgP}$ as pathophysiologically distinct disease $[34,35]$. As a result, a new classification framework for periodontitis has been updated to stages (I-IV) and grades (A-C). Staging is based on the severity and the extent of disease, as well as on management complexity, while grading depends on a history-based analysis of the rate of progression, which is linked with risk factors. As this study started in 2014, a diagnosis of AgP was set as the main inclusion criterion (the new classification was not yet in force). Be that as it may, the stages and grades could have been retrospectively determined using data from patients' medical histories (clinical and radiographic examinations). Consequently, all cases included in this study would be classified as periodontitis stage III (severe periodontitis with potential for additional tooth loss) grade $\mathrm{C}$ (rapid rate of progression), based on the new classification scheme for periodontal diseases. Nonetheless, in this article, the previously accepted definition of $\mathrm{AgP}$ was used for comprehensive reasons [31].

Data on long-term results of GTR in aggressive periodontitis patients are scarce; thus, this study prospectively evaluated and compared two regenerative procedures of intrabony defects in AgP. The reported results showed that the regenerative treatment of intrabony defects in $\mathrm{AgP}$ might lead to positive clinical and radiographic outcomes 4 years after surgery. To the best of our knowledge, this RCT has the longest follow-up available in the literature. Even though high complexity of outcome measures was expressed, tooth loss was a true clinical endpoint. Consequently, one tooth in the control group was lost during the study period due to other than periodontal reasons (root fracture). In general, we observed improvements in the evaluated clinical (PPD reduction, CAL gain) and radiographic (DD reduction, defect fill, bone/graft 
Table 2 Clinical and radiographic parameters: probing pocket depth (PPD), clinical attachment level (CAL), gingival recession (GR), and radiographic defect depth (DD) at the surgical sites at baseline and after 1 and 4 years (mean, 95\% confidence interval (CI), standard deviation (SD))

\begin{tabular}{|c|c|c|c|c|c|c|c|c|}
\hline & \multicolumn{4}{|c|}{ Test sites $(n=15)$} & \multicolumn{4}{|c|}{ Control sites $(n=15)$} \\
\hline & PPD (mm) & CAL (mm) & $\mathrm{GR}(\mathrm{mm})$ & $\mathrm{DD}(\mathrm{mm})$ & PPD (mm) & CAL (mm) & $\mathrm{GR}(\mathrm{mm})$ & $\mathrm{DD}(\mathrm{mm})$ \\
\hline \multicolumn{9}{|l|}{ Baseline } \\
\hline Mean & $7.4^{\mathrm{a}, \mathrm{b}}$ & $8.7^{\mathrm{a}, \mathrm{b}}$ & $1.3^{\mathrm{b}}$ & $5.9^{\mathrm{a}, \mathrm{b}}$ & $7.2^{\mathrm{a}, \mathrm{b}}$ & $8.5^{\mathrm{a}, \mathrm{b}}$ & $1.5^{\mathrm{b}}$ & $5.3^{\mathrm{a}, \mathrm{b}}$ \\
\hline $95 \% \mathrm{CI}$ & $6.5-8.3$ & $7.8-9.6$ & $0.9-1.7$ & $5.2-6.6$ & $6.5-7.9$ & $7.5-9.5$ & $0.8-2.1$ & $4.3-6.3$ \\
\hline SD & 1.5 & 1.6 & 0.7 & 1.2 & 1.3 & 1.8 & 1.1 & 1.8 \\
\hline \multicolumn{9}{|l|}{1 year } \\
\hline Mean & $3.4^{\mathrm{a}}$ & $4.0^{\mathrm{a}}$ & 0.9 & $0.7^{\mathrm{a}}$ & $3.7^{\mathrm{a}}$ & $4.2^{\mathrm{a}}$ & 1.5 & $0.9^{\mathrm{a}}$ \\
\hline $95 \% \mathrm{CI}$ & $2.7-4.0$ & $3.0-4.9$ & $0.3-1.5$ & $0.4-1.0$ & $3.1-4.2$ & $3.5-4.9$ & $0.3-2.8$ & $0.5-1.2$ \\
\hline $\mathrm{SD}$ & 1.1 & 1.6 & 1.1 & 0.9 & 0.9 & 1.2 & 2.2 & 0.6 \\
\hline \multicolumn{9}{|l|}{4 years } \\
\hline Mean & $3.6^{\mathrm{b}}$ & $4.0^{\mathrm{b}}$ & $0.6^{\mathrm{b}}$ & $0.6^{\mathrm{b}}$ & $3.8^{\mathrm{b}}$ & $4.6^{\mathrm{b}}$ & $0.9^{\mathrm{b}}$ & $0.7^{\mathrm{b}}$ \\
\hline $95 \% \mathrm{CI}$ & $2.8-4.4$ & $3.0-5.0$ & $0.1-1.1$ & $0.3-1.0$ & $3.2-4.4$ & $3.9-5.2$ & $0.2-1.6$ & $0.5-0.9$ \\
\hline SD & 1.3 & 1.7 & 0.8 & 0.5 & 1.0 & 1.1 & 1.1 & 0.4 \\
\hline
\end{tabular}

$n$ number of split-mouth defects

${ }^{a}$ Statistically significant difference between baseline and 1 year $(p \leq 0.05)$

${ }^{\mathrm{b}}$ Statistically significant difference between baseline and 4 years $(p \leq 0.05)$

No statistically significant differences were observed between 1 year and 4 years within groups $(p \leq 0.05)$

No statistically significant differences were observed between test and control $(p \leq 0.05)$

density gain) parameters 1 and 4 years after surgery, which were significant in comparison with baseline conditions. However, no significant differences between the groups were found. These results seemed to be stable, since no significant changes were observed from 1 to 4 years postoperatively. In MPM-treated sites, mean CAL gain was found to be $4.8 \mathrm{~mm}$ and mean bone/graft density gain was $90 \%$, as compared with $4.0 \mathrm{~mm}$ and $82 \%$ in CM-treated sites, respectively. Within the limits of the present study, these data suggest that regenerative therapy is a predictable technique that allowed patients diagnosed with $\mathrm{AgP}$ to maintain compromised teeth. However, the reported outcomes did not support a considerable advantage of MPM over CM 4 years following the surgery, although some initial improvements in radiographic outcomes were reported $[15,16]$.

Quite recently Artzi et al. [36] retrospectively evaluated and compared two regenerative modalities of periodontal therapy in individuals with AgP. In that study, GTR-treated sites showed PPD reduction and CAL gain similar to those changes observed in sites treated with the application of enamel matrix derivatives combined with DBBM 1-10-year follow-up. That being said, randomized trials on long-term outcomes of GTR in $\mathrm{AgP}$ are lacking. Thus far, only three RCTs have been published with the follow-up period to 12 months, and therefore, the long-term efficacy of those regenerative therapies remains uncertain. In Rakmanee et al.'s [20, 21] study, SPPF plus CM was compared with SPPF alone. The authors found CAL gain of $1.6 \mathrm{~mm}$, PPD reduction of $2.4 \mathrm{~mm}$, and DD reduction of $2.4 \mathrm{~mm}$ at the GTR sites and CAL gain of $2.1 \mathrm{~mm}$, PPD reduction of $2.5 \mathrm{~mm}$, and DD reduction of $2.2 \mathrm{~mm}$ at the access flap sites 12 months following surgery. Both clinical and radiographic results showed substantial comparability between the two groups. On the other hand, in Queiroz et al.'s [19] research, the use of bone substitute mixed with P-15 without any barrier resulted in a CAL gain and PPD reduction that were comparable to those achieved in the group in which a non-resorbable membrane was placed over intrabony defect. However, the radiographic outcomes were significantly better in the ABM/P-15 group. At the GTR sites, authors reported CAL gain of $2.0 \mathrm{~mm}$, PPD reduction of $2.5 \mathrm{~mm}$, bone fill of $0.7 \mathrm{~mm}$, and bone density gain of $62 \%$, as compared with CAL gain of 1.8, PPD reduction of $2.2 \mathrm{~mm}$, bone fill of $2.4 \mathrm{~mm}$, and bone density gain of $93 \%$ in the ABM/P-15 group. However, the actual success cannot merely be assessed at 6 or 12 months following regenerative treatment, but also has to be evaluated years later. In that regard, the 4-year results of our study compare well with the aforementioned RCTs. Unfortunately, it is not possible to contrast the reported outcomes with others since no other RCT had a follow-up period longer than 12 months.

Even following successful treatment, patients with diagnosed periodontitis require life-long supportive care in order to avoid recurrence of disease [37]. Generally speaking, patients with AgP have significantly better compliance in respect to $\mathrm{ChP}$ patients (57\% vs. $30 \%$ ) [38]. In the present study, all patients were involved in a strict SPT during the first year. 


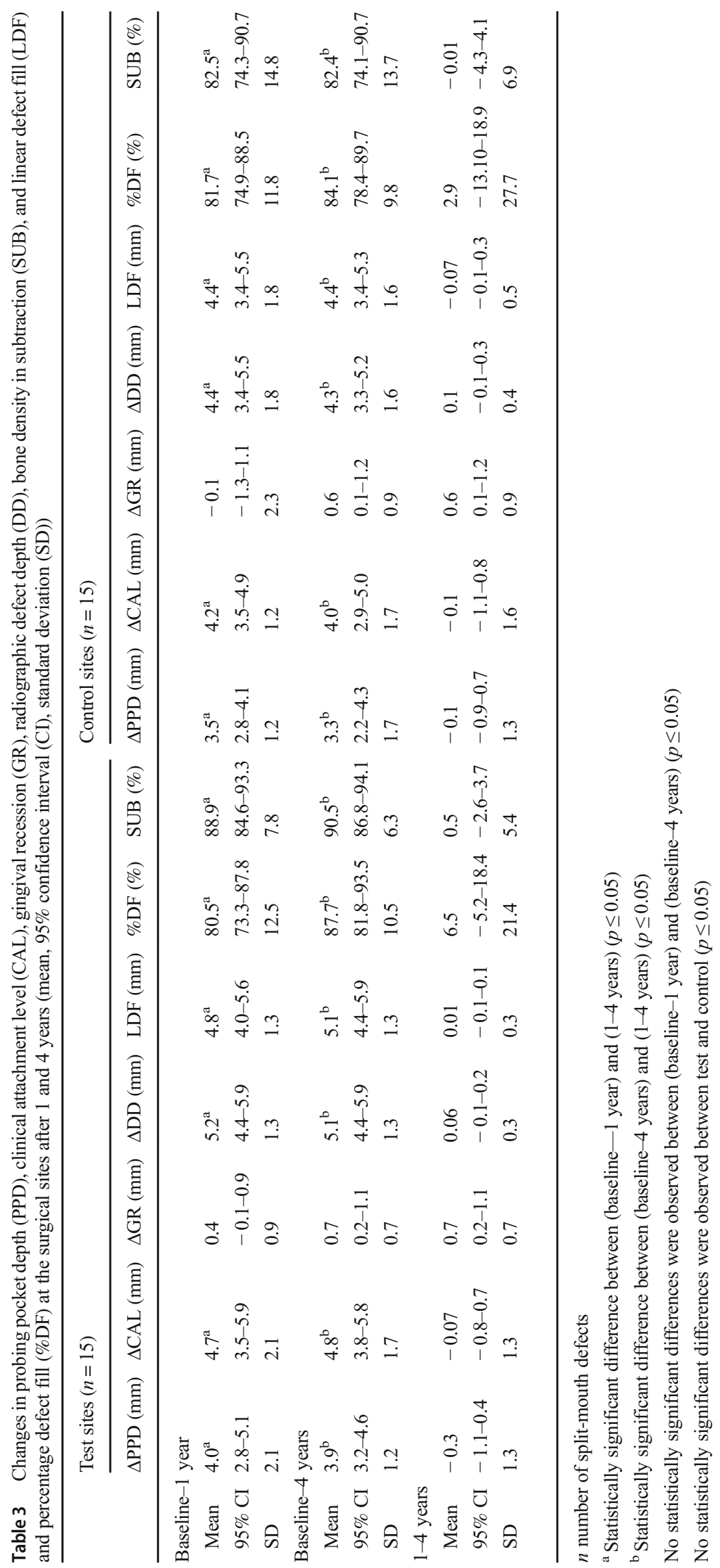


Table 4 Frequency distribution of probing pocket depth $(\mathrm{PPD}) \leq$ $3 \mathrm{~mm}, 4-5 \mathrm{~mm}$ and $\geq 6 \mathrm{~mm}$ at the surgical sites at baseline and after 1 and 4 years

\begin{tabular}{|c|c|c|c|c|c|c|}
\hline & \multicolumn{3}{|c|}{ Test sites $(n=15)$} & \multicolumn{3}{|c|}{ Control sites $(n=15)$} \\
\hline & $\leq 3 \mathrm{~mm}$ & $4-5 \mathrm{~mm}$ & $\geq 6 \mathrm{~mm}$ & $\leq 3 \mathrm{~mm}$ & $4-5 \mathrm{~mm}$ & $\geq 6 \mathrm{~mm}$ \\
\hline Baseline & - & 2 & 13 & - & 1 & 14 \\
\hline 1 year & 8 & 7 & - & 7 & 7 & 1 \\
\hline 4 years & 8 & 4 & 2 & 5 & 7 & 1 \\
\hline
\end{tabular}

$n$ number of split-mouth defects
After that period, further compliance was greatly recommended and 9 patients participated in individually tailored SPT program at the university, but 5 patients returned to referring dentists. FMPS showed only a slightly increasing tendency but remained relatively low throughout the 4-year follow-up (8\% at baseline, $19 \%$ after 1 year, and $26 \%$ after 4 years). Regular participation in SPT and good plaque control have a pivotal impact on long-term success of GTR, as more new attachment loss and tooth loss were found in patients who did not comply with a periodontal maintenance program [39]. In our study, periodontitis recurrence was observed in $22 \%$ of subjects who participated in SPT, and in $40 \%$ who returned to referring dentists. This aspect is of paramount importance, since it seems that regular specialist-based SPT in $\mathrm{AgP}$ patients might act as a protective factor. Multiple linear regression analyses showed that patients who showed disease recurrence exhibited lower CAL gain and bone density gain in the long term. Likewise, the lack of recurrence resulted in $2.94 \mathrm{~mm}$ CAL gain and in almost $10 \%$ bone density gain
4 years following GTR. In consideration of the foregoing, the thorough surgical treatment mode followed by strict supportive periodontal maintenance might be prerequisites for long-time successful results. Due to AgP being a multifactorial and complex disease, the prediction of its progression and response to treatment may differ staggeringly within the same individual and within individuals. By the same token, little is known about the factors that may be used as predictors of therapeutic response to GTR in patients diagnosed with AgP. As a matter of fact, only two long-term interventional studies have assessed prognostic variables among subjects with $\mathrm{AgP}$ who had received active periodontal therapy (APT). One study involving $174 \mathrm{AgP}$ patients demonstrated that smoking and gingival bleeding were associated with an increased risk for periodontitis recurrence 10.5 years following APT [35]. The other found that smoking, stress, and counts of Porphyromonas gingivalis and Treponema denticola were significantly associated with the progressive tissue lost 5 years after initial treatment [40].
Table 5 Regression analysis with clinical attachment level (CAL) gain $(\mathrm{mm})$ from 1 to 4 years as dependent variable. $R^{2}=0.799$

\begin{tabular}{lccccc}
\hline Parameter & $\begin{array}{l}\text { Regression } \\
\text { coefficient }\end{array}$ & Standard Error & \multicolumn{2}{c}{ Confidence interval } & \multirow{2}{*}{$p$ value } \\
\cline { 5 - 6 } & & & Lower & Upper & \\
\hline Intercept & -0.78 & 1.95 & -4.95 & 3.39 & 0.69 \\
Gender & -1.74 & 1.24 & -4.39 & 0.90 & 0.18 \\
Age & -0.02 & 0.02 & -0.07 & 0.02 & 0.25 \\
Surgical procedure (MPM vs. CM) & -0.12 & 0.37 & -0.91 & 0.66 & 0.74 \\
Tooth type (incisors, canines, & 0.20 & 0.66 & -1.20 & 1.61 & 0.76 \\
$\quad$ premolars vs. molars) & & & & & \\
Tooth position (upper vs. lower) & 0.76 & 0.50 & -0.30 & 1.84 & 0.14 \\
Pulp status (vital vs. & -0.03 & 0.55 & -1.22 & 1.15 & 0.95 \\
$\quad$ endodontically treated) & 0.0 & 0.02 & -0.04 & 0.05 & 0.83 \\
FMPS & -0.03 & 0.03 & -0.10 & 0.02 & 0.25 \\
FMBS & 0.07 & 0.24 & -0.45 & 0.59 & 0.77 \\
PPD & 0.20 & 0.20 & -0.22 & 0.64 & 0.32 \\
CAL & -0.46 & 0.57 & -1.69 & 0.76 & 0.43 \\
DD & 0.01 & 0.01 & -0.01 & 0.05 & 0.32 \\
RVG angle & 1.34 & 0.79 & -0.33 & 3.02 & 0.10 \\
Patients' compliance (1 vs. 0) & -2.94 & 0.69 & -4.43 & -1.45 & 0.00 \\
Periodontitis recurrence (1 vs. 0) & & & & & \\
\hline
\end{tabular}

$M P M$ modified perforated membrane, $C M$ collagen membrane, $F M P S$ full-mouth plaque score, $F M B S$ full-mouth bleeding score, $P P D$ probing pocket depth, $D D$ radiographic defect depth 
Table 6 Regression analysis with gain in bone density in subtraction analysis (\%) from 1 to 4 years as dependent variable. $R^{2}=0.653$

\begin{tabular}{llllll}
\hline Parameter & $\begin{array}{l}\text { Regression } \\
\text { coefficient }\end{array}$ & Standard Error & \multicolumn{2}{c}{ Confidence interval } & \multirow{2}{*}{$p$ value } \\
\cline { 3 - 5 } & & & Lower & Upper & \\
\hline Intercept & 1.61 & 10.77 & -21.34 & 24.56 & 0.883 \\
Gender & -8.71 & 6.83 & -23.27 & 5.84 & 0.221 \\
Age & -0.01 & 0.13 & -0.28 & 0.26 & 0.940 \\
Surgical procedure (MPM vs. CM) & 0.43 & 2.03 & -3.91 & 4.76 & 0.837 \\
Tooth type (incisors, canines, & 4.11 & 3.63 & -3.63 & 11.86 & 0.275 \\
$\quad$ premolars vs. molars) & & & & & \\
Tooth position (upper vs. lower) & -3.10 & 2.77 & -9.00 & 2.80 & 0.280 \\
Pulp status (vital vs. endodontically & -1.84 & 3.08 & -8.39 & 4.72 & 0.559 \\
$\quad$ treated) & & & & & \\
FMPS & 0.05 & 0.14 & -0.24 & 0.34 & 0.708 \\
FMBS & -0.22 & 0.17 & -0.58 & 0.14 & 0.213 \\
PPD & -0.05 & 1.34 & -2.91 & 2.82 & 0.973 \\
CAL & 0.63 & 1.12 & -1.76 & 3.02 & 0.583 \\
DD & -2.45 & 3.17 & -9.21 & 4.32 & 0.453 \\
RVG angle & 0.04 & 0.09 & -0.14 & 0.23 & 0.623 \\
Patients' compliance (1 vs. 0) & 9.17 & 4.35 & -0.09 & 18.43 & 0.052 \\
Periodontitis recurrence (1 vs. 0) & -9.66 & 3.85 & -17.85 & -1.46 & 0.024 \\
\hline
\end{tabular}

$M P M$ modified perforated membrane, $C M$ collagen membrane, FMPS full-mouth plaque score, $F M B S$ full-mouth bleeding score, $P P D$ probing pocket depth, $C A L$ clinical attachment level, $D D$ radiographic defect depth
Since the introduction of GTR for the treatment of periodontal defects, continuous improvements have been made in many technical details, as well as in handling of numerous materials [41-43]. The idea behind cellpermeable barrier was for membrane perforations to allow undifferentiated MSCs and growth factors from the surrounding soft tissues to take part in supracrestal regeneration of intrabony defects; thus, molecular migration through the pores might compensate the limited cellularity of the periodontium [9, 11]. Mardas et al. [11] evaluated GTR with demineralized bone matrix and perforated Teflon barriers in rats. Perforations were made utilizing a needle with a diameter of $0.3 \mathrm{~mm}$. After 4 months, the new bone occupied $50.5 \%$ of the cross-sectional area in the test sites and $45.0 \%$ in the sites treated with cellocclusive barrier, but the difference was not statistically significant. On the other hand, Gamal and Iacono [9] suggested modification of collagen membranes. By the same token, MPM had a dense collar that might inhibit epithelial downgrowth on the outer surface and perforated body that may enable PDPCs and GMSCs to migrate into the barrier-protected area and to enhance regenerative processes. As a matter of fact, the superior clinical performance of that device in GTR of intrabony defects in ChP has been confirmed. The postoperative differences between groups were $3.3 \mathrm{~mm}$ and $2.1 \mathrm{~mm}$ for CAL gain, $3.9 \mathrm{~mm}$ and $3.0 \mathrm{~mm}$ for PPD reduction, and $-3 \mathrm{~mm}$ and $-3.1 \mathrm{~mm}$ for DD reduction at 6 months, in favor of the
MPM-treated sites [9]. Moreover, MPM led to enhanced periodontal regeneration in dehiscence defects in dogs with more rapid bone maturation [44]. The impact of MPM on regenerative treatment in $\mathrm{AgP}$ has been reported in the 1-year follow-up of our study [20, 21]. However, with the exception of initially improved LDF (additional $0.4 \mathrm{~mm}, p=0.01$ ), \%DF (additional 7.6\%, $p=0.025$ ), and accelerated bone/graft density (additional 4.9\%, $p=0.011$ ) from 6 to 12 months postoperatively, no significant effects of MPM on the early clinical outcomes were found after 12 months. Similarly, 4 years after surgery, the use of perforated membranes showed no additional benefits yet significant improvements in all evaluated parameters in relation to baseline were observed. Notwithstanding, it has been demonstrated quite recently that MPM may act as non-viral gene delivery devices for bone regeneration protocols [45]. MPM were bioactivated by incorporating plasmid DNA ( $p$ DNA) or chemically modified RNA (cmRNA) encoding bone morphogenetic protein-9 (BMP-9), and their bone regenerating potential was evaluated. Bioactive MPM demonstrated enhanced osteogenic differentiation compared with controls in vitro (higher alkaline phosphatase activity and calcium mineralization) and in vivo using the calvarial defect model in rats (higher distribution of bone volume fraction). This concept has not been tested in intrabony defects in human yet.

The present study is not exempt from limitations. Without doubt, the main disadvantage of this study is that 

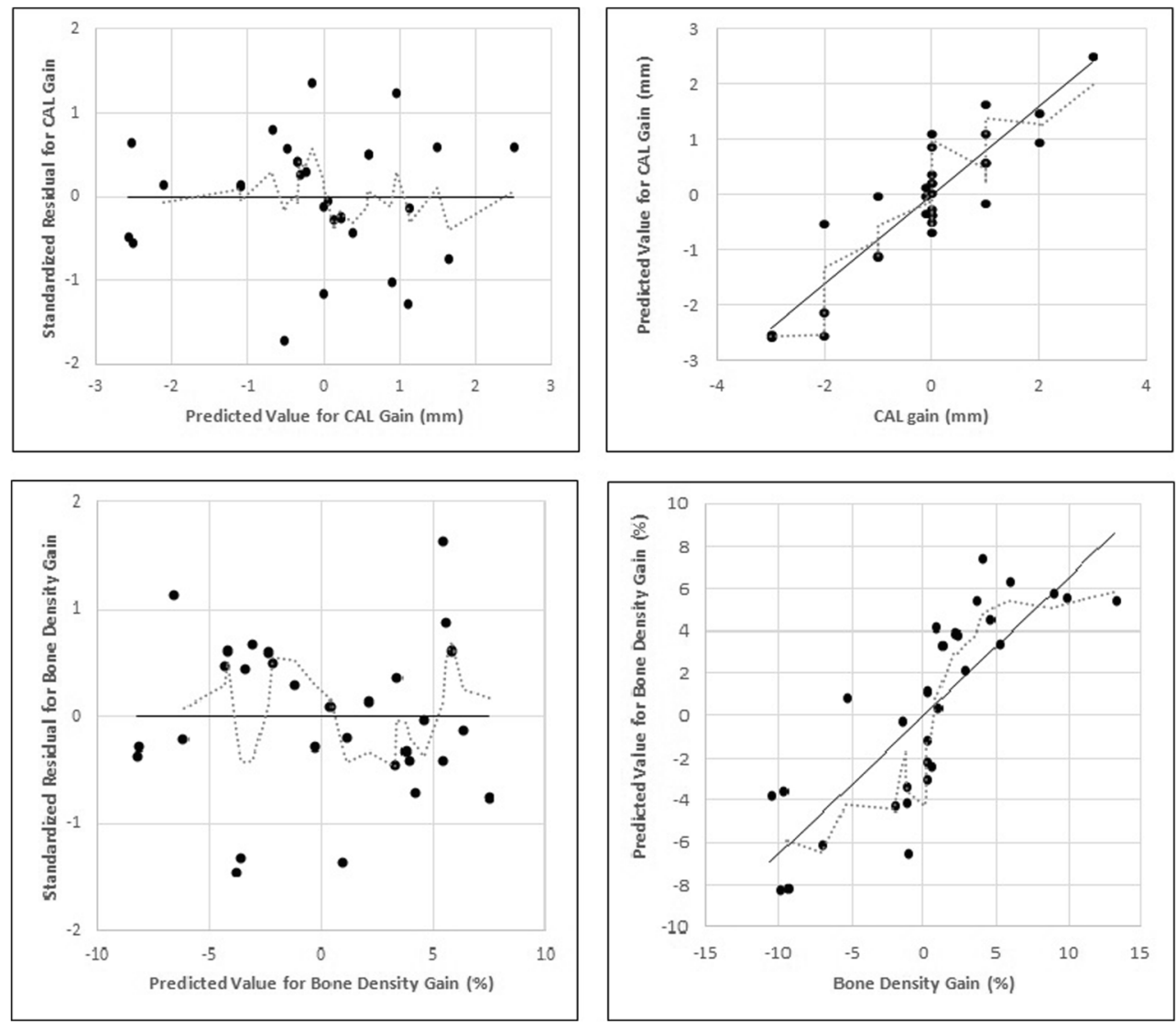

Fig. 4 Scatter diagrams showing the model quality in reference to linearity and homoscedasticity (upper and lower left sides). Scatter diagrams showing the prediction of CAL gain (upper right side) and bone/graft density gain (lower right side) by the regression models

the results obtained are related to a diagnosis of $\mathrm{AgP}$; meanwhile, the current definition of a periodontitis is based on a multi-dimensional staging and grading system. Nevertheless, this research was planned and carried out long before the new classification. Having said that, all patients involved in this study would be classified as periodontitis stage III grade C. Secondly, owing to the relatively small number of subjects, some variables and predictors might not have shown significant associations. Additionally, potential risk of bias due to some drop-outs remained, since one patient did not complete the study, and in another subject, one tooth has been extracted (thus 1 MPM-treated site and $2 \mathrm{CM}$-treated sites were lost to analysis). However, as a consequence of low AgP prevalence in population, other studies have used sample size similar to this of the reported RCT. Moreover, sample size calculation had been carried out before the commencement of this study. Third, we did not perform histological evaluation; hence, the true nature of healing could not be evaluated. DBBM with slow resorption rate was used to fill intrabony defects. Consequently, the observed radiographic fill will consist of a composition of both radiopaque xenogenic graft particles and regenerating vital human bone; thus, DSR may not represent a true bone formation and maturation. Therefore, the histological proof of evidence for true regeneration achieved with MPM should be demonstrated in animal models, as current ethical codes thwart a precise determination of clinical and radiographic improvements in humans. Fourth, albeit from each patient, two, as similar as possible, intrabony defects were selected; there were relatively more maxillary defects in the control group. By any means, the baseline characteristics of defects were controlled to remove the further potential bias caused by the unbalanced baseline values and protection from selection bias was maintained due to randomization and allocation concealment.

All things considered, more research needs to be carried out on the use of MPM in AgP, and the evaluation of longterm stability of achieved clinical and radiographic outcomes is one aspect that requires further investigation. Furthermore, the future design of state-of-the-art biomaterials that will robustly collaborate with self-healing capacity of periodontal defects and enhance specific gingival stem cell regenerative potential requires much more comprehensive insights in the processes underlying biological principles of the cell-based regeneration mechanisms. 


\section{Conclusions}

Within the limitations of this 4-year study, the present findings may imply that:

- GTR of intrabony defects in AgP with either standard or modified CM yielded similarly successful clinical and radiographic benefits for compromised teeth 4 years following the surgery;

- Significant CAL and bone/graft density gains remained stable 4 years after surgery, with minimal changes from 1 to 4 years;

- The use of MPM showed no additional advantage in the long term;

- Periodontitis recurrence was a significant predictor of the clinical response to GTR.

Funding The study was self-supported by authors.

\section{Compliance with ethical standards}

Conflict of interest The authors declare that they have no conflict of interest.

Ethical approval All procedures performed in studies involving human participants were in accordance with the ethical standards of the institutional and/or national research committee and with the 1964 Helsinki declaration and its later amendments or comparable ethical standards. This study was reviewed and approved by the Bioethics Committee of Medical University of Warsaw, Poland (KB/135/2014).

Informed consent Informed consent was obtained from all individual participants included in the study.

Open Access This article is distributed under the terms of the Creative Commons Attribution 4.0 International License (http:// creativecommons.org/licenses/by/4.0/), which permits unrestricted use, distribution, and reproduction in any medium, provided you give appropriate credit to the original author(s) and the source, provide a link to the Creative Commons license, and indicate if changes were made.

\section{References}

1. Jauregui C, Yoganarasimha S, Madurantakam P (2018) Mesenchymal stem cells derived from healthy and diseased human gingiva support osteogenesis on electrospun Polycaprolactone scaffolds. Bioengineering (Basel) 5(1):E8. https://doi.org/10. 3390/bioengineering5010008

2. Filion TM, Skelly JD, Huang H, Greiner DL, Ayers DC, Song J (2017) Impaired osteogenesis of T1DM bone marrow-derived stromal cells and periosteum-derived cells and their differential in-vitro responses to growth factor rescue. Stem Cell Res Ther 8(1):65. https://doi.org/10.1186/s13287-017-0521-6

3. Santamaria S, Sanchez N, Sanz M, Garcia-Sanz JA (2017) Comparison of periodontal ligament and gingiva-derived mesenchymal stem cells for regenerative therapies. Clin Oral Investig 21(4):1095-1102. https://doi.org/10.1007/s00784-016-1867-3
4. Wang YL, Hong A, Yen TH, Hong HH (2019) Isolation of mesenchymal stem cells from human alveolar periosteum and effects of vitamin D on osteogenic activity of periosteum-derived cells. J Vis Exp:135. https://doi.org/10.3791/57166

5. Reynolds MA, Kao RT, Camargo PM, Calton JG, Clem DS, Fiorellini JP, Geisinger ML, Mills MP, Nares S, Nevins ML (2015) Periodontal regeneration- intrabony defects: a consensus report from the AAP Regeneration Workshop. J Periodontol 86(Suppl 2):S105-S107. https://doi.org/10.1902/jop.2015.140378

6. Sculean A, Nikolidakis D, Schwarz F (2008) Regeneration of periodontal tissues: combinations of barrier membranes and grafting materials- biological foundation and preclinical evidence: a systematic review. J Clin Periodontol 35(Suppl 8):106-116. https://doi. org/10.1111/j.1600-051X.2008.01263.x

7. Zellin G, Linde A (1996) Effects of different osteopromotive membrane porosities on experimental bone neogenesis in rats. Biomaterials 17(7):695-702

8. Colnot C, Zhang X, Knothe Tate ML (2012) Current insights on the regenerative potential of the periosteum: molecular, cellular and endogenous engineering approaches. J Orthop Res 30(12):18691878. https://doi.org/10.1002/jor.22181

9. Gamal AY, Iacono VJ (2013) Enhancing guided tissue regeneration of periodontal defects by using a novel perforated barrier membrane. J Periodontol 84(7):905-913. https://doi.org/10.1902/jop. 2012.120301

10. Wikesjö UM, Lim WH, Thomson RC, Hardwick WR (2003) Periodontal repair in dogs: gingival tissue occlusion, a critical requirement for GTR? J Clin Periodontol 30(7):655-664

11. Mardas N, Kostopoulos L, Stavropoulos A, Karring T (2003) Evaluation of a cell-permeable barrier for guided tissue regeneration combined with demineralized bone matrix. Clin Oral Implants Res 14(6):812-818

12. Gamal AY, Al-Berry NN, Hassan AA, Rashed LA, Iacono VJ (2017) In vitro evaluation of the human gingival fibroblast/ gingival mesenchymal stem cell dynamics through perforated guided tissue membranes: cell migration, proliferation and membrane stiffness assay. J Periodontal Res 52(3):628-635. https://doi.org/10.1111/ jre. 12431

13. Gamal AY, Aziz M, Salama MH, Iacono VJ (2014) Gingival crevicular fluid bone morphogenetic protein-2 release profile following the use of modified perforated membrane barriers on localized intrabony defects: a randomized clinical trial. J Int Acad Periodontol 16(2):55-63

14. Gamal AY, Abdel-Ghaffarr K, Iacono VJ (2016) Gingival crevicular fluid vascular endothelial cell growth factor and platelet-derived growth factor-BB release profile following the use of perforated barrier membranes during treatment of intrabony defects: a randomized clinical trial. J Periodontal Res 51(3):407-416. https://doi.org/ $10.1111 /$ jre. 12321

15. Górski B, Jalowski S, Górska R, Zaremba M (2018) Treatment of intrabony defects with modified perforated membranes in aggressive periodontitis: a 12 -month randomized controlled trial. Clin Oral Investig 22(8):2819-2828. https://doi.org/10.1007/s00784018-2368-3

16. Górski B, Jalowski S, Górska R, Zaremba M (2018) Treatment of intrabony defects with modified perforated membranes in aggressive periodontitis: subtraction radiography outcomes, prognostic variables, and patient morbidity. Clin Oral Investig 2018 (in press) 23:3005-3020. https://doi.org/10.1007/s00784-018-2712-7

17. Reynolds MA, Kao RT, Camargo PM, Caton JG, Clem DS, Fiorellini JP, Geisinger ML, Mills MP, Nares S, Nevins ML (2015) Periodontal regeneration - intrabony defects: a consensus report from the AAP RegenerationWorkshop. J Periodontol 86(Suppl 2):S105-S107. https://doi.org/10.1902/jop.2015.140378

18. Corbella S, Weinstein R, Francetti L, Taschieri S, Del Fabbro M (2016) Periodontal regeneration in aggressive periodontitis patients: 
a systematic review of the literature. J Investig Clin Dent 8(4): e12245. https://doi.org/10.1111/jicd.12245

19. Queiroz AC, Nobrega PB, Oliveira FS, Novaes AB Jr, Taba M Jr, Palioto DB, Grisi MF, Souza SL (2013) Treatment of intrabony defects with anorganic bone matrix/p-15 or guided tissue regeneration in patients with aggressive periodontitis. Braz Dent J 24(3): 204-212. https://doi.org/10.1590/0103-6440201302169

20. Rakmanee T, Griffiths GS, Auplish G, Darbar U, Petrie A, Olsen I, Donos N (2016) Treatment of intrabony defects with guided tissue regeneration in aggressive periodontitis: clinical outcomes at 6 and 12 months. Clin Oral Investig 20(6):1217-1225. https://doi.org/10. 1007/s00784-015-1609-y

21. Rakmanee T, Griffiths GS, Auplish G, Darbar U, Petrie A, Olsen I, Donos N (2016) Radiographic outcomes following treatment of intrabony defects with guided tissue regeneration in aggressive periodontitis. Clin Oral Investig 20(6):1227-1235. https://doi.org/10. 1007/200784-015-1609-y

22. Wu YC, Lin LK, Song CJ, Su YX, Tu YK (2017) Comparisons of periodontal regenerative therapies: a meta-analysis on the long-term efficacy. J Clin Periodontol 44(5):511-519. https://doi.org/10.1111/ jcpe. 12715

23. Lang NP, Bartold PM, Cullinan M, Jeffcoat M, Mombelli A, Murakami S, Page R, Papapanou P, Tonetti M, Van Dyke T (1999) Consensus report: aggressive periodontitis. Ann Periodontol 4(1):53. https://doi.org/10.1902/annals.1999.4.1.53

24. O'Leary TJ, Drake RB, Naylor JE (1972) The plaque control record. J Periodontol 43(1):38-46

25. Cortellini P, Pini-Prato G, Tonetti MS (1995) Periodontal regeneration of human infrabony defects I. Clinical measures. J Periodontol 64(4):254-260. https://doi.org/10.1902/jop.1993.64.4.254

26. Eickholz P, Hörr T, Klein F, Hassfeld S, Kim TS (2004) Radiographic parameters for prognosis of periodontal healing of infrabony defects. Two different definitions of defect depth. J Periodontol 75(3):399-407. https://doi.org/10.1902/jop.2004.75. 3.399

27. Jayakumar A, Rajababu P, Rohini S, Butchibabu K, Naveen A, Reddy PK, Vidyasagar S, Satyanarayana D, Pavan Kumar S (2011) Multi-center, randomized clinical trial on efficacy and safety of recombinant human platelet-derived growth factor with $\beta$ tricalcium phosphate in human intra-osseous periodontal defects. J Clin Periodontol 38(2):163-172. https://doi.org/10.1111/j.1600051X.2010.01639.x

28. Cortellini P, Pini-Prato G, Tonetti MS (1995) The modified papilla preservation technique. A new surgical approach for interproximal regenerative procedures. J Periodontol 66(4):261-266. https://doi. org/10.1902/jop.1995.66.4.261

29. Cortellini P, Pini-Prato G, Tonetti MS (1999) The simplified papilla preservation flap. A novel surgical approach for the management of soft tissues in regenerative procedures. Int $\mathrm{J}$ Periodontics Restorative Dent 19(6):589-599

30. Bäumer A, El Sayed N, Kim TS, Reitmeir P, Eickholz P, Pretzl B (2011) Patient-related risk factors for tooth loss in aggressive periodontitis after active periodontal therapy. J Clin Periodontol 38(4): 644-651. https://doi.org/10.1111/j.1600-051X.2011.01698.x

31. Armitage GC (1999) Development of a classification system for periodontal diseases and conditions. Ann Periodontol 22(4):1-6. https://doi.org/10.1902/annals.1999.4.1.1
32. Albandar JM, Tinoco EM (2002) Global epidemiology of periodontal diseases in children and young persons. Periodontol 2000 29: 153-176. https://doi.org/10.1034/j.1600-0757.2002.290108.x

33. Tonetti MS, Mombelli A (2008) Aggressive periodontitis. In: Lindhe J, Karring T, Lang NP (eds) Clinical periodontology and implant dentistry. Oxford, UK, Blackwell Munksgaard, pp 428458

34. Fine DH, Patil AG, Loos BG (2018) Classification and diagnosis of aggressive periodontitis. J Clin Periodontol 45(Suppl 20):S95S111. https://doi.org/10.1111/jcpe.12942

35. Tonetti MS, Greenwell H, Kornman KS (2018) Staging and grading of periodontitis: framework and proposal of a new classification and case definition. J Clin Periodontol 45(Suppl 20):S149-S161. https://doi.org/10.1111/jcpe.12945

36. Artzi Z, Sudri S, Platner O, Kozlovsky A (2019) Regeneration of the periodontal apparatus in aggressive periodontitis patients. Dent J (Basel) 7(1):E29. https://doi.org/10.3390/dj7010029

37. Chapple ILC, Mealey BL, Berglundh T, Chapple ILC, Jepsen S, S Kornman K, L Mealey B, Papapanou PN, Sanz M, S Tonetti M (2018) Periodontal health and gingival diseases and conditions on an intact and a reduced periodontium: consensus report of workgroup 1 of the 2017 World Workshop on the Classification of Periodontal and Peri-implant Diseases and Conditions. J Clin Periodontol 45 (Suppl 20): S1-S8. doi: https://doi.org/10.1111/ jcpe. 12935

38. Agrawal N, Jain R, Jain M, Agarwal K, Dubey A (2015) Compliance with supportive periodontal therapy among patients with aggressive periodontitis and chronic periodontitis. J Oral Sci 57(3):249-254. https://doi.org/10.2334/josnusd.57.249

39. Cortellini P, Tonetti MS (2004) Long-term tooth survival following regenerative treatment of intrabony defects. J Periodontol 75(5): 672-678. https://doi.org/10.1902/jop.2004.75.5.672

40. Kamma JJ, Baehni PC (2003) Five-year maintenance follow-up of early-onset periodontitis patients. J Clin Periodontol 30(6):562572. https://doi.org/10.1034/j.1600-051X.2003.00289.x

41. Gottlow J, Nyman S, Lindhe J, Karring T, Wennström J (1986) New attachment formation in the human periodontium by guided tissue regeneration. J Clin Periodontol 13(6):604-616

42. Nyman S, Lindhe J, Karring T, Rylander H (1982) New attachment following surgical treatment of human periodontal disease. J Clin Periodontol 9(4):290-296

43. Melcher AH (1976) On the repair potential of periodontal tissues. J Periodontol 47(5):256-260

44. Fahmy RA, Kotry GS, Ramadan OR (2018) Periodontal regeneration of dehiscence defects using a modified perforated collagen membrane. A comparative experimental study. Future Dental Journal 4(2):225-230. https://doi.org/10.1016/j.fdj.2018.06.004

45. Khorsand B, Elangovan S, Hong L, Kormann MSD (2018) A bioactive membrane that enhances bone regeneration. J Biomed Master Res B Part B 9999 (9999): 1-9. doi: https://doi.org/10. 1002/jbm.b.34275, 1832

Publisher's note Springer Nature remains neutral with regard to jurisdictional claims in published maps and institutional affiliations. 